\title{
Multiyear statistics of 2-D shortwave radiative effects at three ARM sites
}

\author{
Tamás Várnai
}

University of Maryland Baltimore County, Joint Center for Earth System Technology

Prepared for Journal of the Atmospheric Sciences

March 2010 


\begin{abstract}
This study examines the importance of horizontal photon transport effects, which are not considered in the 1-D calculations of solar radiative heating used by most atmospheric dynamical models. In particular, the paper analyzes the difference between 2-D and 1-D radiative calculations for 2-D vertical cross-sections of clouds that were observed at three sites over 2- to 3-year periods. The results show that 2-D effects increase multiyear 24-hour average total solar absorption by about $4.1 \mathrm{~W} / \mathrm{m}^{2}, 1.2 \mathrm{~W} / \mathrm{m}^{2}$, and $0.3 \mathrm{~W} / \mathrm{m}^{2}$ at a tropical, mid-latitude, and arctic site, respectively. However, 2-D effects are often much larger than these average values, especially for high sun and for convective clouds. The results also reveal a somewhat unexpected behavior, that horizontal photon transport often enhances solar heating even for oblique sun. These findings underscore the need for fast radiation calculation methods that can allow atmospheric dynamical simulations to consider the inherently multidimensional nature of shortwave radiative processes.
\end{abstract}




\section{Introduction}

Because of the prohibitive computational demands of three-dimensional (3-D) radiative calculations, most atmospheric dynamical simulations use onedimensional (1-D) radiation models to calculate solar heating. However, several studies have indicated that the inherently multidimensional nature of cloud radiative processes can cause large errors in 1-D calculations (e.g., 0'Hirok and Gauthier 1998, 2005; Di Giuseppe and Tompkins 2003, 2005; Pincus et al. 2005). Complementing the detailed case studies, 2-D radiative calculations using the $4 \mathrm{~km}$ resolution clouds of a monthlong global simulation also showed some significant departures from 1-D theory (Cole et al. 2005). But even though such studies provided many valuable insights, it remains unclear how important the multidimensional nature of solar radiative processes is for atmospheric simulations.

The US Department of Energy ARM Climate Research Facility (ACRF) offers excellent opportunities to address this issue by providing long-term detailed cloud observations at several sites. At these sites, ground-based vertically pointing instruments provide 2-D vertical cross-sections through the clouds drifting aloft. By comparing the results of 1-D and 2-D radiation simulations for clouds observed at a tropical, mid-latitude, and arctic location, this paper provides some initial estimates on the typical magnitude of 2-D radiative effects. Because the calculations don't include cross-wind cloud variability, the presented 2-D effects can be considered as conservative, lower-bound estimates for the full 3-D effects (Pincus et al. 2005). 


\section{Dataset and methodology}

We analyze three years of cloud data at ACRF's NSA (Northern Slope of Alaska) and SGP (Southern Great Plains) sites, from 2005-2007 and 1999-2001, respectively. At the TWP (Tropical Western Pacific) site we analyze the two years (2003-2004) for which Microbase data is available for the full year. The ACRF Microbase product combines millimeter-wavelength cloud radar, micropulse lidar, and microwave radiometer data to estimate cloud liquid and ice water content and particle size at $10 \mathrm{~s}$ temporal and $45 \mathrm{~m}$ vertical resolution. These time-dependent vertical profiles are converted into 2-D spatial structures using each cloud layer's mean wind speed from the ACRF Mergesonde product and the frozen turbulence assumption (e.g., Barker et al. 2004). Though the actual resolution of the obtained 2D fields varies with the wind speed (median values: $86 \mathrm{~m}$ at NSA, $141 \mathrm{~m}$ at SGP, and $74 \mathrm{~m}$ at TWP), for the radiative calculations we resample all data to a uniform $50 \mathrm{~m}$ resolution.

While the ACRF Microbase and Mergesonde products are certainly not perfect and are influenced by both instrument uncertainties and retrieval algorithm limitations, they arguably represent the current state-of-the-art for comprehensive, long-term, and detailed cloud structure datasets. This analysis considers all daytime data, but excludes $10 \mathrm{~km}$ wide swaths around data gaps and around data flagged with a low quality control flag.

We simulate radiative transfer through the observed clouds using a forward Monte Carlo model that was tested in the I3RC project (Cahalan et al., 2005) and 
through comparisons to broadband SBDART (Ricchiazzi et al. 1998) calculations. The 2-D simulations assume no variability in the cross-wind direction and-to capture as much of the 3-D radiative process as possible-they assume that the solar azimuth is parallel to the wind (e.g., Várnai and Marshak 2003, Pincus et al., 2005). We perform two 2-D simulations for each scene, with the sun on the downwind and upwind sides, respectively.

Because the goal is to obtain robust statistics on the difference between 1-D and 2-D results-as opposed to obtaining accurate results for individual cloudsthe simulations can use both relatively few photons (up to about 3000 for each $50 \mathrm{~m}$ column), and moderately complex radiative characteristics. Minor imperfections are not expected to influence the obtained statistics because the 1-D and 2-D simulations use the same Monte Carlo radiative transfer model and setup.

The Monte Carlo model calculates gaseous absorption in the $0.2-5.0 \mu \mathrm{m}$ range using the correlated-k method, with coefficients from SBDART (Ricchiazzi et al. 1998). The SBDART tropical atmospheric profile is used for the TWP site, and the mid-latitude summer (winter) and sub-arctic summer (winter) profiles are used for the SGP and NSA sites during the April-September (October-March) periods, respectively. Cloud particle scattering and absorption parameters are from Mie calculations for liquid droplets, and from the publicly available database of Baum (http://www.ssec.wisc.edu/ baum/Cirrus/Solar_Spectral_Models.html) for ice crystals. Rayleigh scattering is considered, but aerosol effects are not included.

Since Barker and Davies (1992) shows that the angular pattern of surface reflection has only a modest influence on 3-D radiative effects, all simulations use 
Lambertian surfaces with $5 \mathrm{~nm}$-resolution spectral albedos. The sea surface albedos used at the TWP site are from the database described in Jin et al. (2004). The used albedo values depend on the local mean cloud optical thickness and solar elevation, and assume a $5 \mathrm{~m} / \mathrm{s}$ wind speed. Surface albedos for the SGP and NSA sites are specified by the SBDART model's "vegetation", "sand", and "snow" surface types. At SGP, the surface type is selected daily based on human observer reports about surface conditions (documented in ACRF's Surflog product), with the "sand" type used for days without snow or green vegetation. At NSA, where no such data is available, "vegetation" is used from June to September, and "snow" for the rest of the year. These dates were selected based on snow cover data from the Rutgers University Global Snow Lab (http://climate.rutgers.edu/snowcover/).

\section{Results}

We examine the influence of 2-D radiative effects by comparing the results of 1-D and 2-D radiative simulations. Table 1 presents the influence of 2-D effects on multiyear 24-hour average solar radiative fluxes (including cloud-free periods and nighttime as well). The table shows that 2-D effects are stronger at lower latitudes, due to factors such as

- more intense solar illumination,

- stronger convection creating more heterogeneous clouds, 
- more effective channeling process for high sun, guiding photons from opaque to thin regions where they can reach the surface easier (e.g., Davis and Marshak 2001).

We note that the 2-D effects in Table 1 may be considered as conservative, lower-bound estimates for the full 3-D radiative effects, because earlier case studies found 3-D effects to be about 30\% stronger than 2-D effects (e.g., Pincus et al. 2005). The difference arises because 2-D calculations don't consider the cross-wind cloud variability (perpendicular to the solar azimuth) that further reduces cloud reflection and enhances surface absorption through additional channeling. We also note that in coarse-resolution cloud simulations, the errors of 1-D albedo and surface absorption calculations are further increased by the plane-parallel bias caused by unresolved small-scale cloud variability (e.g., Cahalan et al. 1994). As a final note, we also mention that for reflective surfaces, the influence of 2-D effects is somewhat larger on downwelling fluxes at the surface than on surface absorption.

Figure 1 shows the way average 2-D effects depend on solar zenith angle. 2-D effects being largest for high sun means that they are strongest near noon and during the summer. The figure also shows that for relatively high sun, 2-D effects are nearly as strong at NSA as at SGP, which implies that Alaska clouds in the summer and near-noon are almost as heterogeneous as Oklahoma clouds in the spring or fall, or in the morning or afternoon.

The figure also shows that 2-D effects increase atmospheric absorption even for high sun. This may be explained by cloud side illumination increasing the sunlight intercepted by clouds, and also by the trapping of sunlight in thicker cloud 
portions where it is more likely to get absorbed (e.g., O'Hirok and Gauthier 1998; Várnai and Davies 1999).

A somewhat unexpected feature of Figure 1 is a behavior opposite to the dominant 2-D effect discussed in most earlier case studies (e.g., McKee and Cox, 1974) where, for low sun, cloud sides intercepting extra sunlight enhanced reflectivity and reduced transmission to the surface. Figure 1 indicates that 2-D effects often enhance surface absorption even for oblique sun. As Figure 2 illustrates, this occurs when the incoming direct sunlight slips obliquely under an extensive cloud and, after reflection from the surface, gets trapped by the cloud above (O’Hirok and Gauthier 1998).

Naturally, 2-D effects also depend on cloud type. We examine this issue using the ACRF cloud classification product (Cldclass) available at the SGP site (Wang and Sassen, 2001). Figure 3 shows that 2-D effects tend to be strongest for the more heterogeneous, convective cloud types. The main exception is surface absorption near deep convective clouds, because these highly reflective clouds greatly reduce surface absorption in both 1-D and 2-D simulations.

While average values can be informative, detailed histograms can also help in evaluating the importance of 2-D effects. Figure 4 shows that local 2-D effects are often much larger than the average values discussed above. For example at TWP, 2-D effects change the total cloud absorption of $1 \mathrm{~km}$-size columns by more than $20 \%$ in about a third of cases, and they change the total surface absorption of $10 \mathrm{~km}$-size areas by more than $50 \mathrm{~W} / \mathrm{m}^{2}$ in about a quarter of cases. 


\section{Summary}

This study presents multiyear statistics on the influence of 2-D shortwave radiative effects that are not considered in the 1-D radiation calculations used by most atmospheric dynamical models. The influence of 2-D effects on solar radiative energy budget calculations is presented for a tropical, mid-latitude, and arctic site of the US Department of Energy ARM Climate Research Facility. The results show that:

- 2-D effects increase 24-hour average total solar absorption by about $4.1 \mathrm{~W} / \mathrm{m}^{2}$ at the TWP site in Papua-New Guinea, by $1.2 \mathrm{~W} / \mathrm{m}^{2}$ at the SGP site in Oklahoma, and by $0.3 \mathrm{~W} / \mathrm{m}^{2}$ at the NSA site in Alaska.

- 2-D effects are often much larger than these average values, especially for high sun and for convective clouds. Such variations in 2-D effects can change the spatial and temporal distribution of solar radiative heating.

- 2-D effects often increase solar heating even for oblique sun. This occurs when the incoming direct sunlight slips obliquely under an extensive cloud, and the cloud subsequently intercepts much of the light reflected from the surface.

The presented 2-D effects can be considered as conservative, lower-bound estimates for the influence of horizontal photon transport not included in 1-D radiation calculations, because 2-D calculations don't consider cross-wind cloud variability. In earlier case studies the full 3-D effects exceeded 2-D effects by about $30 \%$ (e.g., Pincus et al. 2005). We note that the upcoming installation of scanning radars at ACRF sites will offer new opportunities for fully 3-D studies. 
The results imply that considering horizontal photon transport can greatly improve both the interpretation of shortwave radiative flux measurements and the solar heating calculations in atmospheric dynamical models. This underscores the need for fast radiation calculation methods that can allow dynamical models to consider the inherently three-dimensional nature of shortwave radiative processes.

\section{Acknowledgments}

Financial support from the US Department of Energy Atmospheric System Research program is gratefully acknowledged. Thank you to M. Dunn for providing large sets of Microbase data, and to R. F. Cahalan, K. F. Evans, J. Y. Harrington, and A. Marshak for encouragement and fruitful discussions. 


\section{References}

Barker, H. W., and J. A. Davies, 1992: Solar radiative fluxes for broken cloud fields above reflecting surfaces. J. Atmos. Sci., 49, 749-761.

Barker, H. W., C. F. Pavloski, M. Ovtchinnikov, E. E. Clothiaux, 2004: Assessing a cloud optical depth retrieval algorithm with model-generated data and the frozen turbulence assumption. J. Atmos Sci., 61, 2951-2956.

Cahalan, R. F., L. Oreopoulos, A. Marshak, K. F. Evans, A. B. Davis, R. Pincus, K. Yetzer, B. Mayer, R. Davies, T. Ackerman, H. Barker, E. Clothiaux, R. Ellingson, M. Garay, E. Kassianov, S. Kinne, A. Macke, W. OHirok, P. Partain, S. Prigarin, A. Rublev, G. Stephens, F. Szczap, E. Takara, T. Várnai, G. Wen, and T. Zhuravleva, 2005: The International Intercomparison of 3D Radiation Codes (I3RC): Bringing together the most advanced radiative transfer tools for cloudy atmospheres. Bull. Amer. Meteor. Soc., 86, 1275-1293.

Cahalan, R. F., W. Ridgway, W. J. Wiscombe, T. L. Bell, and J. B. Snider, 1994: The albedo of fractal stratocumulus clouds. J. Atmos. Sci., 51, 2434-2455.

Cole, J. N. S., H. W. Barker, W. O'Hirok, E. E. Clothiaux, M. F. Khairoutdinov, and D. A. Randall, 2005: Atmospheric radiative transfer through global arrays of 2D clouds. Geophys. Res. Lett., 32, L19817, doi:10.1029/2005GL023329.

Davis, A. B., and A. Marshak, 2001: Multiple scattering in clouds: Insights from threedimensional diffusion/P 1 theory. Nucl. Sci. Eng., 137, 251-280.

Di Giuseppe, F., and A. M. Tompkins, 2005: Impact of cloud cover on solar radiative biases in deep convective regimes. J. Atmos. Sci., 62, 1989-2000. 
Di Giuseppe, F., and A. M. Tompkins, 2003: Three dimensional radiative transfer in tropical deep convective clouds. J. Geophys. Res., 108, 10.1029/2003JD003392.

Jin, Z., T. Charlock, W. Smith Jr., and K. Rutledge, 2004: A parameterization of ocean surface albedo. Geophys. Res. Lett., 31, L22301, doi:10.1029/2004GL021180.

McKee, T. B., and S. K. Cox, 1974: Scattering of visible radiation by finite clouds. J. Atmos. Sci., 31, 1885-1892.

0'Hirok, W., and C. Gauthier, 2005: The impact of model resolution on differences between independent column approximation and Monte Carlo estimates of shortwave surface irradiance and atmospheric heating rate. J. Atmos. Sci., 62, 2939-2951.

O'Hirok, W., and C. Gauthier, 1998: A three-dimensional radiative transfer model to investigate the solar radiation within a cloudy atmosphere. Part I: Spatial effects. J. Atmos. Sci., 55, 2162-2179.

Pincus, R., C. Hannay, and K. F. Evans, 2005: The accuracy of determining threedimensional radiative transfer effects in cumulus clouds using ground-based profiling instruments. J. Atmos. Sci., 62, 2284-2293.

Ricchiazzi, P., S. Yang, C. Gautier, and D. Sowle, 1998: SBDART: A research and teaching software tool for plane-parallel radiative transfer in the Earth's atmosphere. Bull. Amer. Meteor. Soc., 79, 2101-2114.

Várnai, T., and A. Marshak, 2003: A method for analyzing how various parts of clouds influence each other's brightness. J. Geophys. Res., 108, 4706, doi: 10.1029/2003JD003561. 
Várnai, T., and R. Davies, 1999: Effects of cloud heterogeneities on shortwave radiation: Comparison of cloud top variability and internal heterogeneity. J. Atmos. Sci., 56, 4206-4224.

Wang, Z. and K. Sassen, 2001: Cloud type and macrophysical property retrieval using multiple remote sensors. J. Appl. Meteor., 40, 1665-1682. 


\section{Tables}

Table 1. Multiyear 24-hour average 2-D effects on shortwave radiative fluxes, including nighttime and clear areas as well. The values indicate the difference between 2-D and 1-D fluxes simulated at full resolution. Uncertainties are standard errors based on the spread of results when the full dataset is divided into 25 subsets. These standard errors likely underestimate actual uncertainties, because they assume that the 25 subsets are independent from each other.

\begin{tabular}{|c|c|c|c|}
\hline Site & $\begin{array}{c}\text { TOA reflected flux } \\
\left(\mathbf{W} / \mathbf{m}^{\mathbf{2}}\right)\end{array}$ & $\begin{array}{c}\text { Atmospheric } \\
\text { absorption }\left(\mathbf{W} / \mathbf{m}^{\mathbf{2}}\right)\end{array}$ & $\begin{array}{c}\text { Surface absorption } \\
\left(\mathbf{W} / \mathbf{m}^{\mathbf{2}}\right)\end{array}$ \\
\hline \hline TWP & $-4.10 \pm 0.21$ & $1.47 \pm 0.03$ & $2.63 \pm 0.17$ \\
\hline SGP & $-1.21 \pm 0.05$ & $0.52 \pm 0.02$ & $0.68 \pm 0.05$ \\
\hline NSA & $-0.28 \pm 0.04$ & $0.25 \pm 0.01$ & $0.02 \pm 0.04$ \\
\hline
\end{tabular}


Figures
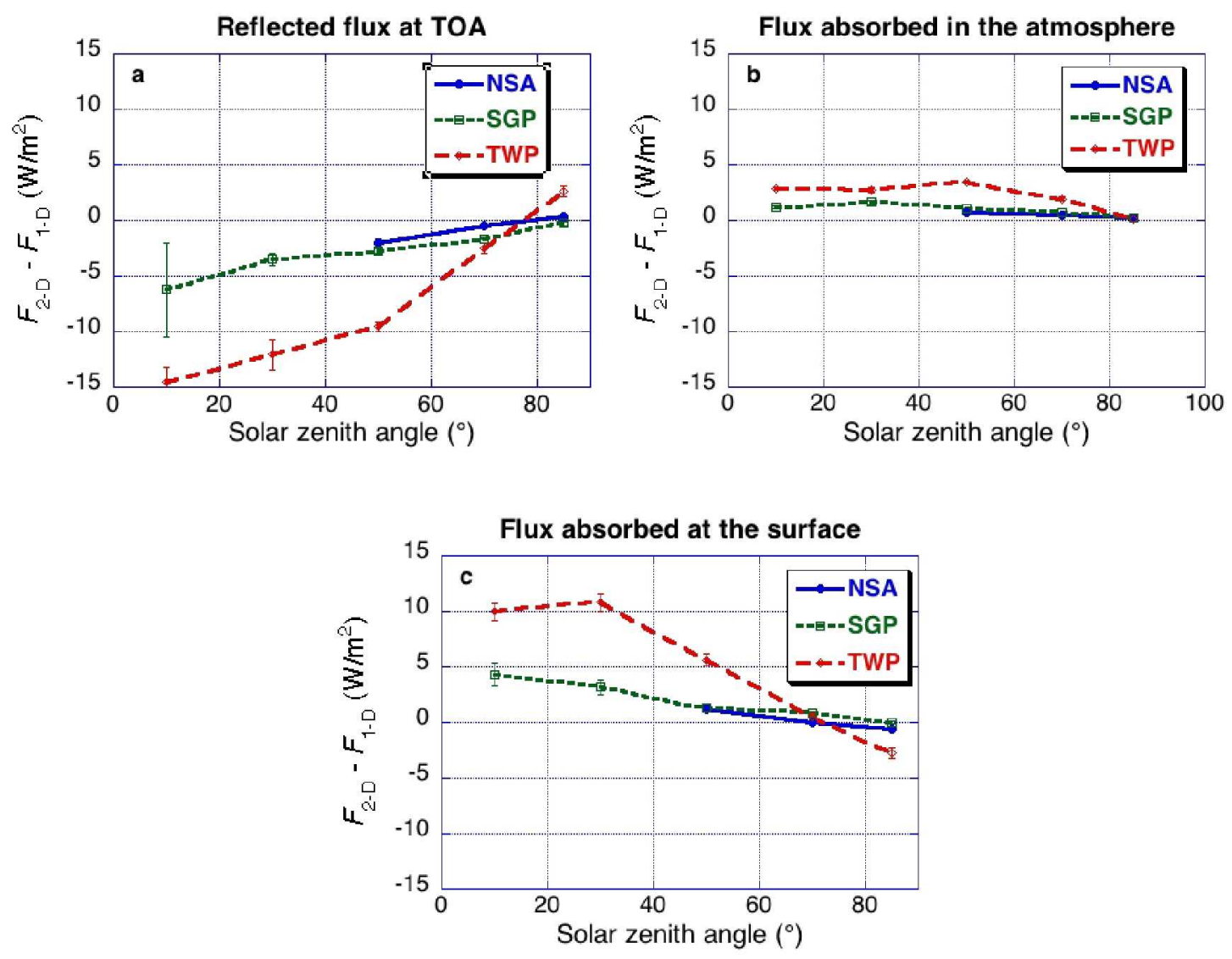

Figure 1. Average 2-D effects on radiative fluxes, as a function of solar zenith angle. (a) Reflected flux; (b) Flux absorbed in the atmosphere; (c) Flux absorbed at the surface. Error bars in this and subsequent figures show standard errors based on the spread of results when the full dataset is divided into 25 subsets. These standard errors likely underestimate actual uncertainties, because they assume that the 25 subsets are independent from each other. 


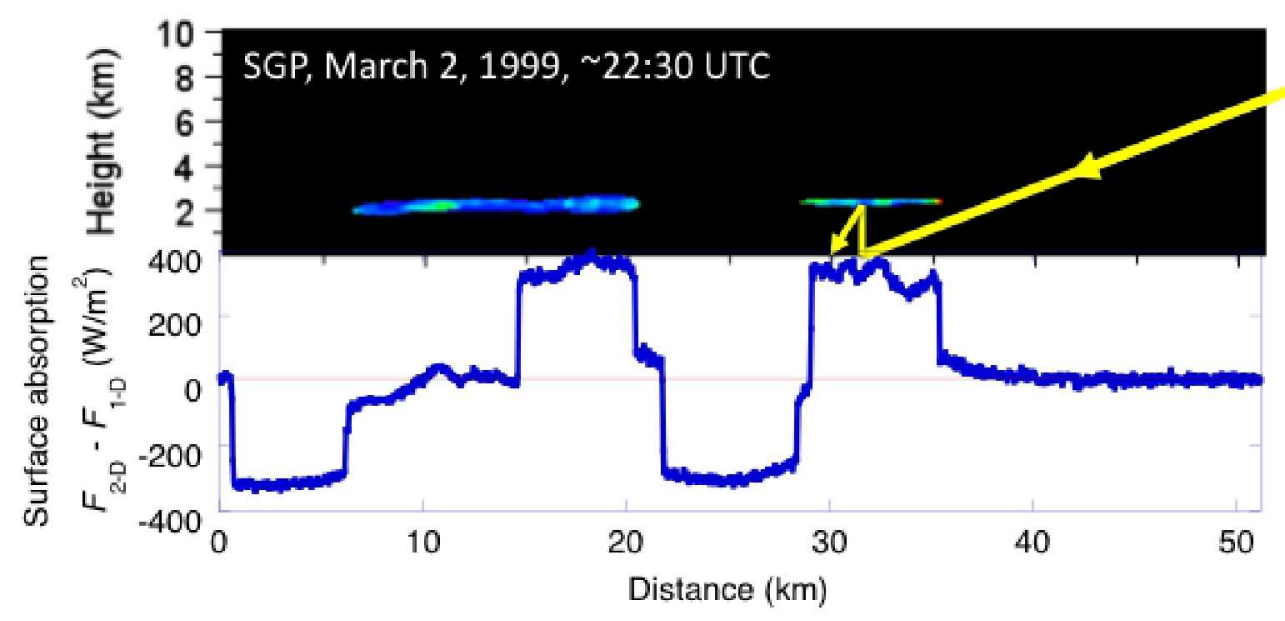

Figure 2. Illustration of the photon trapping process that enhances surface absorption for oblique sun. (top) Illustration using an observed field of volume extinction coefficients; (bottom) Influence of 2-D effects on simulated surface absorption values. 2-D effects enhance the scene average surface absorption by $9.6 \mathrm{~W} / \mathrm{m}^{2}$. The solar zenith angle is $69^{\circ}$. 


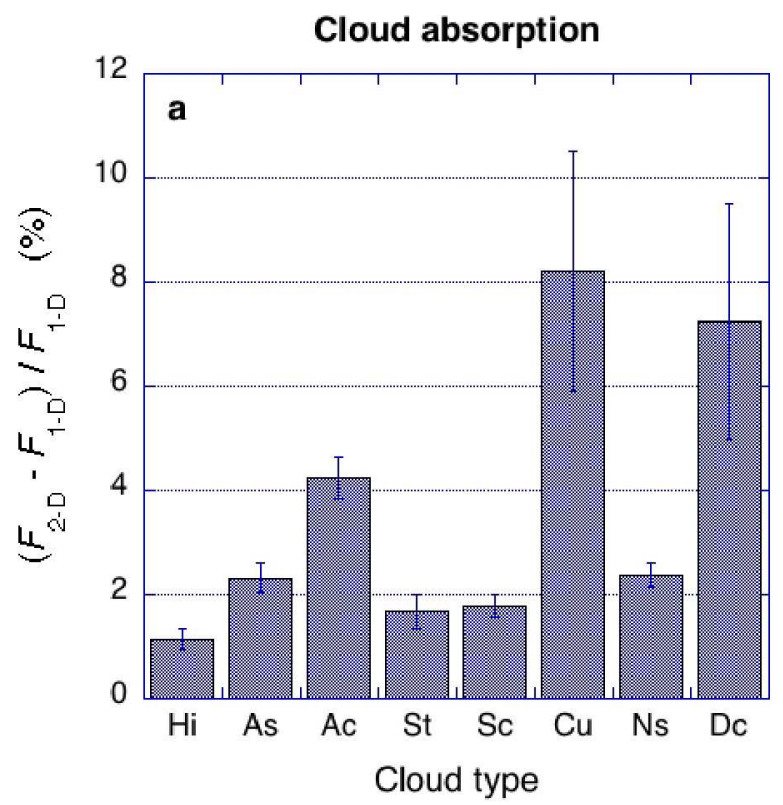

Surface absorption within $10 \mathrm{~km}$ of clouds

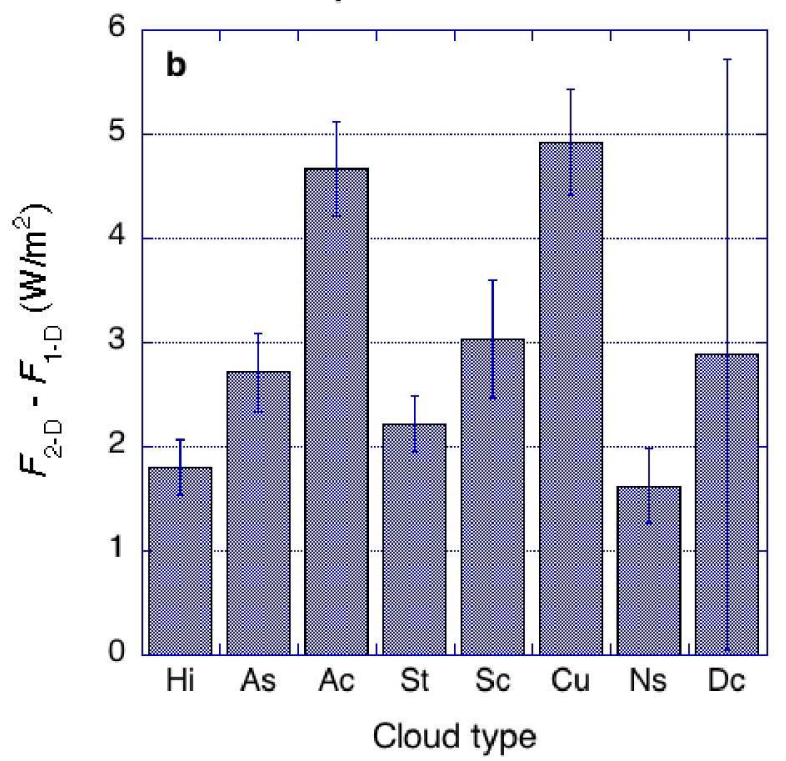

Figure 3. Average 2-D radiative effects for various cloud types at the SGP site. (a) Relative change in cloud absorption; (b) Absolute change in daytime average surface absorption within $10 \mathrm{~km}$ from clouds of each type $\left(\mathrm{W} / \mathrm{m}^{2}\right)$. 


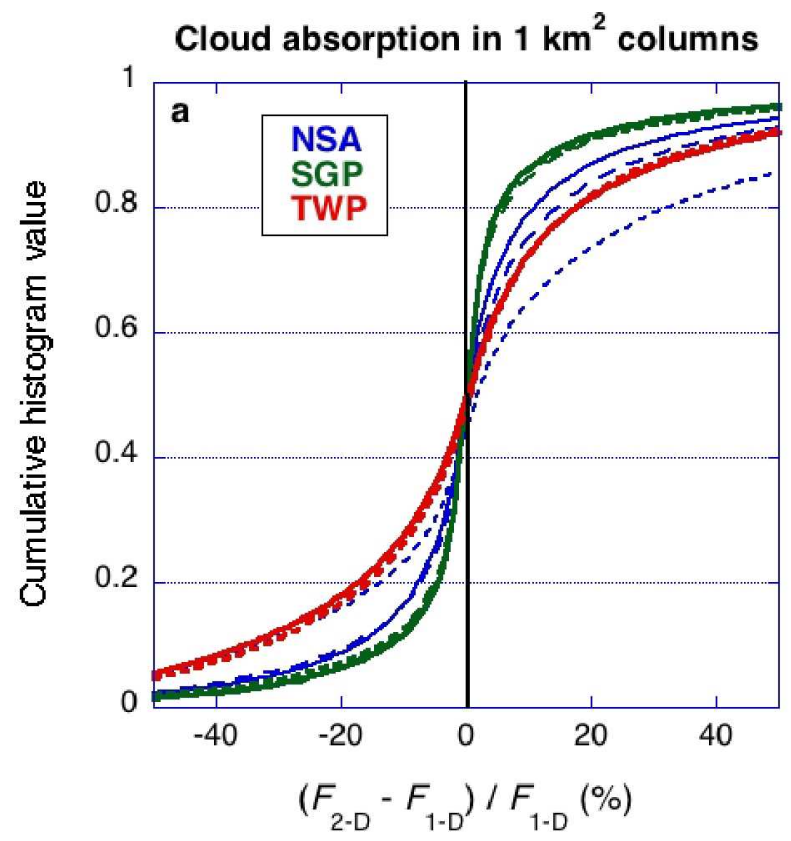

Surface absorption in $\mathbf{1 0} \mathbf{~ k m}$ areas

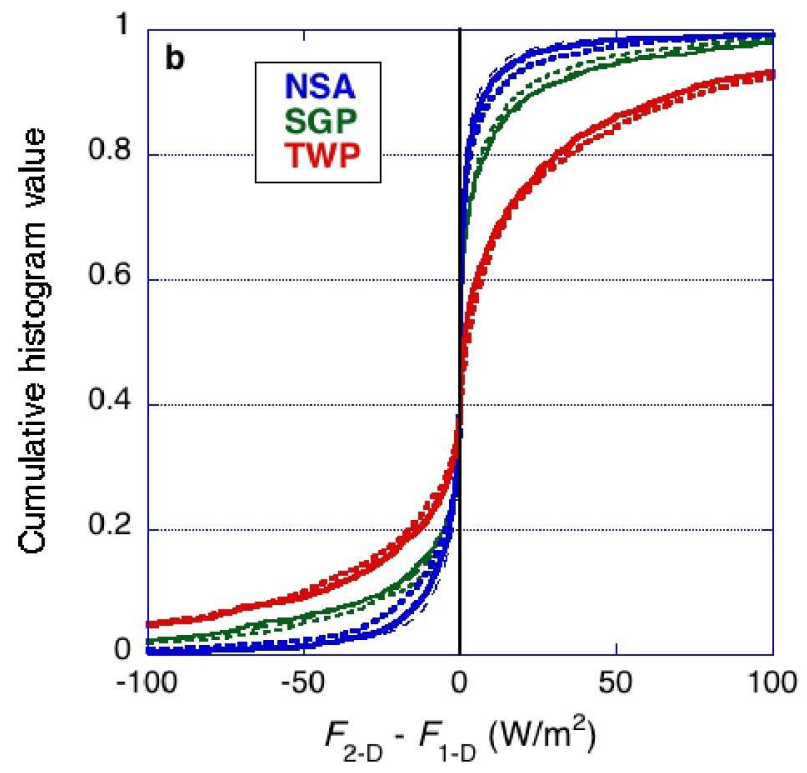

Figure 4. Cumulative histogram of 2-D effects on radiative fluxes. Each curve is for a different year. (a) Relative change in the total absorption of $1 \mathrm{~km}$-size columns; (b) Absolute change in the daytime average surface absorption of all $10 \mathrm{~km}$-size areas, including clear regions. 